
O. Bastien ${ }^{1}$
D. Kerboeuf 2
F. Leimbacher ${ }^{1}$
J. Gevrey ${ }^{3}$
J.A. Nicolas 4
J. Hubert ${ }^{2}$,
O. Heinrich 1

\section{Recherche des causes d'échecs thérapeutiques de la lutte contre les strongyloses gastro-intestinales des} lovins en Martinique

BASTIEN (O), KERBOEUF (D.), LEIMBACHER (F.), GEVREY (J.), NICOLAS (J.A), HUBERT (J.), HEINRICH (O.)BGRPOAß. Recherche des causes d'échecs thérapeutiques dans la lutte contre les strongyloses gastro-intestinales des ovins en Martinique. Revue Elev. Méd. vét. Pays trop., 1991 ( $^{\circ}$ spécial) : 117-121.

L'inefficacité des anthelminthiques a été constatée dans certains élevages ovins de Martinique. Elle paraît due à des pratiques défectueuses des vermifugations et à une gestion inadéquate des pâtures ayant induit l'apparition d'une résistance des strongles aux dérivés du benzimidazole. Les conditions tropicales propices au développement des strongles, la rapidité des réinfestations, la fréquence excessive de traitements $\alpha 2 \alpha$ réalisés avec le même produit utilisé de façon prolongée, sont des facteurs susceptibles d'avoi favorisé la sélection de souches de strongles résistantes. Mots clés: Ovin - Strongle - Résistance au médicament - Benzimidazole - Gestion du pâturage - Martinique.

\section{INTRODUCTION}

Parmi les maladies parasitaires affectant les petits ruminants, les strongyloses gastro-intestinales jouent un rôle important. En milieu tempéré, elles sont dues à plusieurs genres de strongles, généralement associés chez le même animal.

Une enquête épidémiologique, effectuée sur plusieurs années, a révélé les particularités de ces maladies en Martinique (10). La fréquence et l'incidence du parasitisme sur les productions ovines et caprines sont particulièrement élevées du fait du climat tropical. Comme en Guadeloupe (7), le parasite largement dominant est Haemonchus contortus. Ce nématode de la caillette est très pathogène et très prolifique. Son cycle de développement est extrêmement rapide quand le milieu lui est favorable (chaleur et humidité). Sa survie peut être longue, même dans des conditions qui sont néfastes aux autres parasites (4). II supporte bien les températures élevées (14) et les réhydratations suivant les périodes de sécheresse $(13,15)$. II est donc particulièrement bien adapté au milieu tropical.

\section{ITOVIC, Habitation Bonne-Mère, 97224 Ducos, Martinique \\ 2. INRA, Tour nouzilly, 37380 Monnaie. \\ 3. École Nationale vétérinaire, 69752 Charbonnières.}

4. Laboratoire départemental, Avenue du Pr Joseph de Léobardy, 87000 Limoges.
Une prophylaxie rigoureuse est indispensable pour lutter contre cette parasitose. Elle doit théoriquement associer l'utilisation d'anthelminthiques et une gestion adéquate des pâtures. Cette deuxième condition est rarement respectée. D'autre part, les produits utilisés jusqu'à présent chez les petits ruminants n'ont pas d'action rémanente. De ce fait, dans les jours qui suivent le traitement, les animaux peuvent se réinfester s'ils sont placés sur une parcelle contaminée. L'éleveur est alors obligé de multiplier les traitements et peut ainsi favoriser la sélection de parasites résistants aux anthelminthiques.

TABLEAU I Pratique de la vermifugation dans les élevages de l'enquête.

\begin{tabular}{|c|c|c|c|c|}
\hline $\begin{array}{c}\text { Élevage } \\
n^{\circ}\end{array}$ & $\begin{array}{l}\text { Localisation } \\
\text { géographique }\end{array}$ & $\begin{array}{l}\text { Anthelminthique } \\
\text { utilisé } \\
\text { (nbre d'années } \\
\text { d'utilisation) }\end{array}$ & $\begin{array}{c}\text { Fréquence } \\
\text { des } \\
\text { traitements }\end{array}$ & $\begin{array}{l}\text { Qualité } \\
\text { de la } \\
\text { vermi- } \\
\text { fugation }\end{array}$ \\
\hline 1 & Carbet (N) & IVM (3) & $1 M$ & * \\
\hline 2 & Ste Anne (S) & FBZ (5) IVM (2) & $1 \mathrm{M}(2 \mathrm{M}) \mathrm{a}$ & $\star \star *$ \\
\hline 3 & Ste Anne (S) & FBZ (5) & $3 \mathrm{M}$ & ** \\
\hline 4 & Ste Anne (S) & FBZ (5) & $1 \mathrm{M}$ & *** \\
\hline 5 & Ste Anne (S) & FBZ (5) & irrégulière & * \\
\hline 6 & Ste Anne (S) & FBZ (5) & $2 \mathrm{M}$ & * \\
\hline 7 & Petit Bourg (C) & FBZ (5) & $1 \mathrm{M}$ & ** \\
\hline 8 & Ducos $(C)$ & FBZ (5) IVM (3) & $1 \mathrm{M}$ & *** \\
\hline 9 & François (C) & FBT (3) & $1 \mathrm{M}$ & * \\
\hline 10 & Morne-Rouge (N) & FBZ (5) IVM (3) & $1 \mathrm{M}$ & $\star \star \star *$ \\
\hline 11 & Trinité (N) & FBZ (5) & $1 \mathrm{M}$ & $* * *$ \\
\hline 12 & Ste Anne (S) & FBZ (5) & $2 \mathrm{M}$ & * \\
\hline 13 & Ste Anne (S) & $\begin{array}{l}\text { FBZ (5) FBT (2) } \\
\text { IVM (1) }\end{array}$ & irrégulière & ** \\
\hline 14 & Ste Anne (S) & FBZ (5) & $2 M$ & * \\
\hline 15 & Marin (S) & FBZ (5) OXZ (1) & $1 \mathrm{M}$ & $\star \star \star *$ \\
\hline 16 & Marin (S) & OXZ (5) & $1 \mathrm{M}$ & ** \\
\hline 17 & Diamant (S) & $0 \times Z(5)$ & irrégulière & * \\
\hline 18 & Diamant (S) & $0 \times Z(5)$ & $1 \mathrm{M}$ & 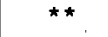 \\
\hline 19 & Diamant (S) & $0 \times 2(5)$ & $1 \mathrm{M}$ & $\star \star \star *^{\prime}$ \\
\hline 20 & Fort-de-France $(\mathrm{C})$ & $0 \times Z(5)$ & $1 \mathrm{M}$ & $* * *$ \\
\hline
\end{tabular}

(N) $=$ nord $\quad F B T=$ Fébantel (Rintal N.D. $)$

(C) $=$ centre $\quad F B Z=$ Fenbendazole (Panacur N.D. $)$

(S) = sud $\quad$ IVM $=$ Ivermectine (IVomec N.D. $)$ OXZ = Oxtendazole (Synanthic N.D.)

$1 \mathrm{M}=$ mensue $2 \mathrm{M}=$ bimensuel $3 \mathrm{M}=$ trimestriel

a - fréquence d'utilisation do l'ivcrmectine

* nombreuses erreurs de pratique

** = quelques erreurs

** = bonne pratique de vermifugation 
O. Bastien, D. Kerboeuf, F. Leimbacher, J. Gevrey, J.A. Nicolas, J. Hubert, O. Heinrich

Des échecs thérapeutiques signalés à partir de 1984 en Martinique ont conduit à la mise en place d'une étude portant sur leur réalité et leurs causes. II était indispensable de faire la part des échecs dus à une mauvaise pratique de la vermifugation de ceux dus à des phénomènes de résistance, sans oublier que les erreurs de stratégie antiparasitaire peuvent aussi conduire à la sélection de parasites résistants.

L'enquête a tenu compte de tous ces aspects. Elle s'est déroulée en deux étapes:

- au cours de la première, les conditions de réalisation des vermifugations ont été analysées;
- la deuxième partie a eu pour objectif de rechercher l'existence de résistance et de la quantifier. Ce travail a été effectué selon le schéma décrit précédemment (3) qui comprend deux parties: détermination de la présence de strongles résistants dans l'élevage par examens coproscopiques avant et après traitement avec le produit suspecté, mesure de l'intensité de la résistance par tests in vitro.

De l'ensemble des travaux réalisés $(2,12)$, il ressort un certain nombre de faits qui permettent dès à présent de faire quelques recommandations relatives à l'usage des anthelminthiques en Martinique.

TABLEAU II Classification des élevages en fonction des résultats des examens coproscopiques effectués avant et après traitement.

\begin{tabular}{|c|c|c|c|c|c|c|}
\hline \multirow{3}{*}{$\begin{array}{c}\text { Élevage } \\
n^{\circ}\end{array}$} & \multicolumn{5}{|c|}{ Résultats des examens coproscopiques } & \multirow{3}{*}{$\begin{array}{l}\text { Classifi- } \\
\text { cation }\end{array}$} \\
\hline & \multicolumn{2}{|c|}{ Avant traitement } & \multicolumn{3}{|c|}{ Après traitement } & \\
\hline & Nbre de positifs & Opg moyen & Substance & Nbre de positifs & Opg moyen & \\
\hline $\begin{array}{l}1 \\
2(\operatorname{lot} 1) \\
(\operatorname{lot} 2)\end{array}$ & $\begin{array}{l}18^{*} \\
16 \\
10\end{array}$ & $\begin{array}{r}1350 \\
806 \\
215\end{array}$ & $\begin{array}{l}\text { IVM } \\
\text { IVM } \\
\text { IVM }\end{array}$ & $\begin{array}{l}0^{*} \\
0 \\
0\end{array}$ & $\begin{array}{l}0 \\
0 \\
0\end{array}$ & $\begin{array}{l}\text { témoins } \\
\text { AH de } \\
\text { référence }\end{array}$ \\
\hline 3 & 10 & 185 & FBZ & 0 & 0 & $\begin{array}{l}\text { témoin } \\
\text { "sensible " }\end{array}$ \\
\hline $\begin{array}{r}4 \\
5 \\
6 \\
7 \\
8 \\
9 \\
10 \\
11\end{array}$ & $\begin{array}{r}14 \\
19 \\
18 \\
14 \\
9 \\
19 \\
11 \\
12\end{array}$ & $\begin{array}{r}669 \\
695 \\
795 \\
414 \\
340 \\
+542 \\
750 \\
3996\end{array}$ & $\begin{array}{l}\text { FBZ } \\
\text { FBZ } \\
\text { FBZ } \\
\text { FBZ } \\
\text { FBZ } \\
\text { FBT } \\
\text { FBZ } \\
\text { FBZ }\end{array}$ & $\begin{array}{l}1 \\
3 \\
4 \\
2 \\
0 \\
4 \\
0 \\
0\end{array}$ & $\begin{array}{r}500 \\
250 \\
200 \\
100 \\
0 \\
138 \\
0 \\
0 \\
\end{array}$ & $\begin{array}{c}\text { élevages } \\
\text { non: } \\
\text { " résistants " }\end{array}$ \\
\hline $\begin{array}{l}2 ' \text { * * } \\
11 \\
12 \\
13 \\
14 \\
15 \\
16 \\
17 \\
18 \\
19 \\
20\end{array}$ & $\begin{array}{r}19 \\
15 \\
13 \\
18 \\
10 \\
16 \\
18 \\
18 \\
16 \\
7\end{array}$ & $\begin{array}{r}680 \\
-610 \\
1108 \\
1228 \\
290 \\
1580 \\
597 \\
1626 \\
1015 \\
535\end{array}$ & $\begin{array}{l}\text { FBZ } \\
\text { FBZ } \\
\text { FBZ } \\
\text { FBT } \\
\text { FBZ } \\
\text { OFZ } \\
\text { FBZ } \\
\text { FBZ } \\
\text { FBZ } \\
\text { FBZ } \\
\text { FBZ }\end{array}$ & $\begin{array}{r}16 \\
12 \\
11 \\
9 \\
16 \\
5 \\
8 \\
13 \\
16 \\
16 \\
10\end{array}$ & $\begin{array}{r}1035 \\
216 \\
1495 \\
572 \\
512 \\
590 \\
1006 \\
173 \\
512 \\
1143 \\
520\end{array}$ & $\begin{array}{c}\text { élevages } \\
\text { "tolérants" } \\
\text { ou } \\
\text { "résistants " }\end{array}$ \\
\hline
\end{tabular}

$\mathrm{AH}=$ anthelminthique

non "résistants " = nombre de positifs inférieur à 2

ou $\left\{\begin{array}{l}\text { excrétion d'œufs nulle ou faible }(<500) \text { après traitement } \\ \text { et nombre de positifs }(n): 2<n<5\end{array}\right.$

"résistants" ou " tolérants" = nombre de positifs supérieur à 5

FBZ = Fenbendazole (Panacur N.D.)

FBT = Fébantel (Rintal N.D.)

IVM = Ivermectine (lvomec N.D.)

OFZ = Oxfendazole (Synanthic N.D.)

* sur 20 animaux

** $2^{\mathrm{E}}$ série de prélèvements. 


\section{Pratique de la vermifugation}

La première partie de l'enquête a confirmé la réalité des échecs thérapeutiques (12). Les erreurs compromettant l'efficacité des traitements ont été recensées.

L'absence de méthode et de dispositif pour le rassemblement des animaux est fréquemment constatée. L'adaptation de la dose au poids de l'agneau ou de la brebis est rarement réelle : les éleveurs ne pèsent pas leurs animaux et ne les séparent pas en catégories de poids identiques. Pour tenter de remédier à ces erreurs, une plaquette rappelant les principes de la lutte contre les parasites internes des ovins et des caprins a été conçue et diffusée par l'ITOVIC (9).

D'autre part, les recontaminations immédiatement après le traitement sont fréquentes: la plupart des éleveurs ne disposent pas de pâtures indemnes de parasites et, après la vermifugation, remettent ou laissent leurs animaux sur des parcelles contaminées.

\section{Mise en évidence de cas de résistance aux helminthiques}

Les renseignements recueillis au cours de la première partie de l'étude (12) n'ont pas apporté d'indications précises quant à l'existence de phénomènes de résistance mais ils ne permettaient pas non plus d'en écarter la possibilité. En particulier, la présence de strongles résistants aux dérivés du noyau benzimidazole était suspectée dans certains élevages du fait de l'importance du parasitisme et des pratiques de vermifugation.

TABLEAU III Valeur des DL 50 et des facteurs de résistance $(F . R .=D L 50$ «souche testée $/ / D L 50$ «souche sensible de référence $\gg)$.

\begin{tabular}{|c|c|c|c|}
\hline $\begin{array}{c}\text { Élevage } \\
\mathrm{n}^{\circ}\end{array}$ & $\begin{array}{r}\text { DL 50 } \\
(\mu \mathrm{g} / \mathrm{ml})\end{array}$ & F.R. & classement \\
\hline 3 & 0,033 & - & " sensible " \\
\hline 15 & 0,155 & 4,7 & " tolérant " \\
\hline $2^{*}$ & 2,067 & 62,6 & \\
$11^{*}$ & 2,963 & 90,8 & \\
13 & 2,122 & 64,3 & \\
14 & 3,168 & 96,0 & \\
16 & 6,220 & 187,2 & " résistants " \\
17 & 1,535 & 46,5 & \\
18 & 2,996 & 90,8 & \\
19 & 2,910 & 88,2 & \\
20 & 5,362 & 162,5 & \\
\hline
\end{tabular}

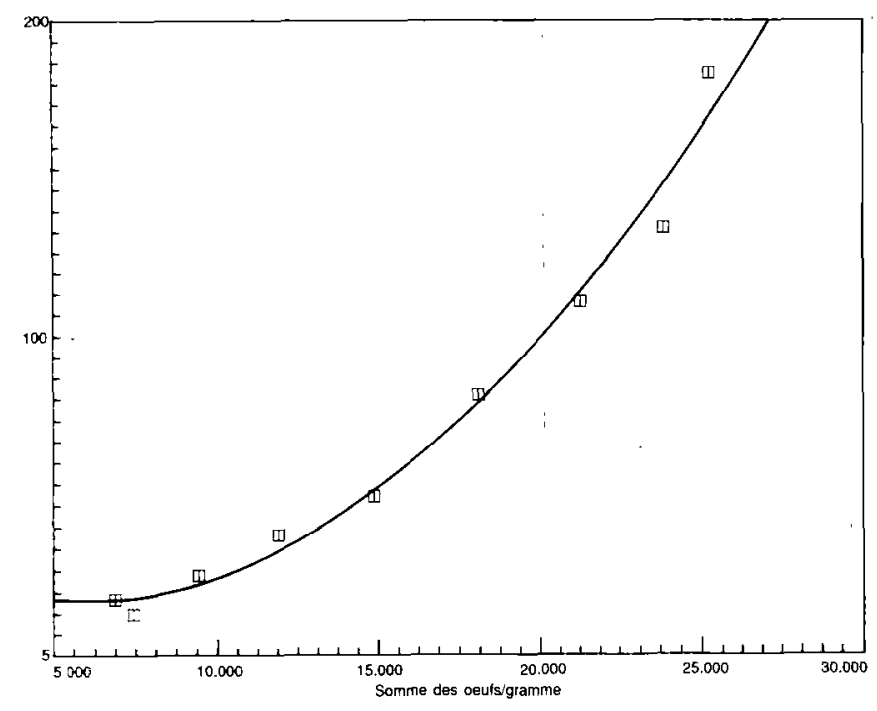

Fig. I: Facteur de résistance

Les facteurs favorisant le développement de phénomènes de résistance ne sont pas encore bien identifiés (8). II semble que la multiplication des traitements, l'utilisaton prolongée du même produit ou de la même famille de produits, la vermifugation en période de faible recontamination, le sous-dosage augmentent la vitesse de sélection des souches résistantes.

Plusieurs de ces conditions sont souvent réunies en Martinique où l'on constate :

- une très grande fréquence des traitements: mensuelle dans la presque totalité des élevages ;

- une utilisation quasi-exclusive des anthelminthiques appartenant à la famille des dérivés du noyau benzimidazole: fenbendazole dans la grande majorité des cas, oxfendazole parfois ;

- une faible fréquence des alternances avec d'autres produits.

Par contre, certaines conditions devraient plutôt retarder le développement de la résistance (8) :

- les recontaminations importantes après traitement sont quasiment la règle ;

- il n'existe pas de sous-dosage, au moins volontaire. Au contraire, beaucoup d'éleveurs augmentent la posologie par crainte de manque d'efficacité.

Les résultats obtenus (2) ont révélé l'existence de parasites résistants dans neuf des vingt élevages objet de l'enquête. Le calcul des facteurs de résistance (FR), qui permettent de comparer les parasites étudiés aux souches normalement sensibles au produit testé, a montré que la résistance est particulièrement élevée $(F R=45$ à 190). 
O. Bastien, D. Kerboeuf, F. Leimbacher, J. Gevrey, J.A. Nicolas, J. Hubert, O. Heinrich

TABLEAU IV Résultats des bilans parasitaires. Tous ont été effectués sur des agneaux sauf celui de l'élevage Trinité, sur brebis.

\begin{tabular}{|c|c|c|c|c|c|c|c|c|}
\hline \multirow{2}{*}{ Nombre de vers } & \multicolumn{3}{|c|}{ Élevages " sensibles" } & \multicolumn{5}{|c|}{ Élevages " résistants" } \\
\hline & Ste Anne & \multicolumn{2}{|c|}{ Ste Anne (a) et (b) } & \multicolumn{2}{|c|}{ Trinité (a) et (b) } & Ste Anne & \multicolumn{2}{|c|}{ Diamant (a) et (b } \\
\hline Haemonchus contortus & & a & $b$ & a & $b$ & \multirow[b]{2}{*}{5625} & a & $b$ \\
\hline femelles & 4000 & 150 & 3375 & 1000 & 105 & & 820 & 380 \\
\hline - vulve avec languette & 64,6 & \multicolumn{2}{|c|}{60,6} & \multicolumn{2}{|c|}{64,5} & 69,2 & \multicolumn{2}{|c|}{74,2} \\
\hline — vulve " boutonnée" & 23,2 & \multicolumn{2}{|c|}{18,6} & \multicolumn{2}{|c|}{0,0} & 14,5 & \multicolumn{2}{|c|}{12,9} \\
\hline - vulve lisse & 12,2 & \multicolumn{2}{|c|}{20,8} & \multicolumn{2}{|c|}{35,5} & 16,3 & \multicolumn{2}{|c|}{12,9} \\
\hline mâles & 2925 & 125 & 2600 & 1375 & 0 & 2900 & 1240 & 320 \\
\hline Total & 6925 & 275 & 5475 & 2375 & 105 & 8525 & 2060 & 700 \\
\hline
\end{tabular}

La sélection de strongles résistants, appartenant au genre Haemonchus, paraît devoir être attribuée essentiellement à l'utilisation quasi-exclusive de substances dérivées du noyau benzimidazole (fenbendazole, oxfendazole) à un rythme mensuel ou bimensuel depuis environ cinq ans. La sélection semble pouvoir s'effectuer très rapidement, en l'espace de quelques mois. La réversion paraît difficile à obtenir, même en utilisant d'autres produits.

Le rôle d'autres facteurs, tels que le type d'élevage, la localisation dans l'île et le mode de conduite du troupeau, n'a pu être mis en évidence.

\section{CONCLUSION}

Ces travaux confirment l'existence de strongles résistants aux anthelminthiques dans les élevages martiniquais $(5,6)$.

Ce phénomène est un des facteurs limitants de l'efficacité des vermifugations. Certaines erreurs dans le choix des produits, en particulier l'emploi quasiexclusif d'un seul groupe d'anthelminthiques (benzi- midazoles), dans les rythmes de traitement et dans la réalisation des vermifugations ont, sans doute, favorisé le développement de ce phénomène.

Le simple remplacement de ces produits par d'autres ne peut suffire à l'éradication de la résistance. En effet, l'utilisation exclusive, dans les mêmes conditions, d'une autre substance est susceptible de conduire rapidement à la sélection de parasites résistants à plusieurs familles.

Pour limiter le développement du phénomène, l'emploi alterné de substances ayant des modes d'action différents est préconisé, l'intervalle de changement étant défini de telle façon qu'une même génération de strongles ne soit pas soumise aux produits successivement utilisés (11). Le délai entre chaque génération de parasite reste à déterminer. Cela suppose une connaissance plus appronfondie de la biologie des écotypes des espèces de strongles présentes en Martinique. Des informations plus précises relatives à la survie et au développement de ces parasites permettraient d'autre part de proposer, en association aux traitements, des systèmes de gestion des pâturages mieux adaptés. Les vermifugations pourraient alors être espacées et la pression de sélection exercée sur des populations résistantes serait ainsi réduite. De tels systèmes ont été proposés pour les bovins en Guadeloupe (1). 
BASTIEN (O.), KERBOEUF (D.), LEIMBACHER (F.), GEVREY (J.), NICOLAS (J.A), HUBERT (J.), HEINRICH (O.)BGRPOA $\beta$. Research of therapeutic inefficacy reasons in the control of sheep gastro-intestinal strongylosis in Martinique. Revue Elev. Méd. vét. Pays trop., 1991 ( $\mathrm{n}^{\circ}$ spécial) : 117-121.

Inefficacy of anthelmintics has been observed in some ovine flocks of Martinique. It seems to be due to defective vermifugation practices and to inadequate pasture managements, responsibles of the emergence of a resistance of gastro-intestinal parasites to benzimidazoles. The tropical conditions are favorable for the development of parasites, the infestations are very fast. The treatments are made with an excessive frequency, with the same compound, used for a long period, all factors able to induce the selection of strains of helminths resistant to chemicals. Key words : Sheep - Strongles - Drug resistance - Benzimidazole - Vermifugation - Pasture management - Martinique.

\section{BIBLIOGRAPHIE}

1. AUMONT (G.). Dynamique des populations de stades infestants de strongles gastro-intestinaux en Guadeloupe (FWI) : conséquences épidémiologiques de différents types de gestion des pâturages. In : L'alimentation des ruminants en milieu tropical humide. Paris, INRA, 1988. (Les Colloques de l'INRA).

2. BASTIEN (O.), KERBOEUF (D.), LEIMBACHER (F.), GEVREY (J.), NICOLAS (J.A.), HUBERT (J.). Mise en évidence de strongles résistants aux benzimidazoles dans les élevages ovins de la Martinique. Recl. Méd. vét., 1989, 165 (5) : 461-467.

3. BEAUMONT-SCHWARTZ (C.), KERBOEUF (D.), HUBERT (J.). Méthodes de mise en évidence de souches de strongles gastro-intestinaux résistantes aux anthelminthiques. Recl. Méd. vét., 1987, 163 : 683-688.

4. BULLICK (G.R.), ANDERSEN (F.L.). Effect of irrigation on survival of third stage Heamonchus contortus larvae (Nematoda : Trichostrongyloidea). Great Basin Naturalist, 1978, $38: 368-378$.

5. GRUNER (L.). Contrôle des strongyloses digestives des petits ruminants aux Antilles françaises : développement de résistance aux benzimidazoles et intérêt d'une gestion raisonnée des pâturages. Revue Elev. Méd. vét. Pays trop., 1985, 38 (4) : 386-393.

6. GRUNER (L.), KERBOEUF (D.), BEAUMONT (C.), HUBERT (J.). Résistance to benzimidazoles of Haemonchus contortus utkalensis in sheep on Martinique. Vet. Rec., 1986, $118: 276$.

7. GRUNER (L.), PEROUX (F.), AUMONT (G.). Dynamique des populations de parasites internes dans un élevage semi-intensif de chèvres créoles en Guadeloupe. In : Les maladies de la chèvre, Niort, 1984. Paris, INRA, P. 695-704 (Les colloques de $1^{\prime}$ INRA $n^{\circ} 28$ ).

8. KERBOEUF (D.). La résistance des strongles aux anthelminthiques : données générales. Revue Méd. vét., 1988, 139 : 6167.

9. LEIMBACHER (F.), DELAVENAY (R.), FOUQUET (E.). Comment lutter contre les parasites internes. Conseils pour bien vermifuger ovins et caprins. 1987, Fiche ITOVIC.

10. LEIMBACHER (F.), LIABEUF (J.M.). Précisions sur la nature, l'importance économique et l'épidémiologie des principales maladies rencontrées chez les ovins et les caprins aux Antilles françaises. Les maladies de la chèvre, Niort, 9-11 oct. 1984. Paris, INRA, P. 731-742. (Les Colloques de l'INRA n 28).

11. LE JAMBRE (L.F.). Anthelmintic resistance in gastro-intestinal nematodes of sheep. In: DONALD (A.D.), SOUTHCOTT (XW.H.), DINFFN (J.K.), ed. The epidemiology and control of gastro-intestinal parasites of sheep in Australia. CSIRO, Div. Anim. Hlth, Australia, 1978. P. 109-120.

12. MALIALIN (F.), LEIMBACHER (F.). Etude sur le déparasitage interne des ovins et caprins à la Martinique. Compterendu d'enquête préliminaire. Paris, ITOVIC, 1986. 18 p.

13. TODD (K.S.), LEVINE (N.D.), BOATMAN (P.A.). Effect of dessication on the survival of infective Haemonchus contortus larvae under laboratory conditions. J. Parasit., 1976, 62: 247-249.

14. TODD (K.S.), LEVINE (N.D.), BOATMAN (P.A.). Effect of temperature on the survival of free-living stages of Haemonchus contortus. Am. J. vet. Res., 1976, 37 : 991-992.

15. TODD (K.S.), LEVINE (N.D.), WHITESIDE (C.C.). Moisture stress effects on survival infective Haemonchus contortus larvae. J. Nematol, $1970,2: 330-333$. 